\title{
Societal systems - complex or worse?
}

\author{
Claes Andersson \\ Complex Systems Group, Division for Physical Resource Theory, Department of Energy and \\ Environment, Chalmers University of Technology, 41296 Göteborg, Sweden. \\ Anton Törnberg \\ Department of Sociology and Work Science, University of Gothenburg, Box 720, 40530 \\ Göteborg, Sweden. \\ Petter Törnberg \\ Complex Systems Group, Division for Physical Resource Theory, Department of Energy and \\ Environment, Chalmers University of Technology, 41296 Göteborg, Sweden.
}

\begin{abstract}
The basic observation that we explore in this paper is simple but, we argue, rich in consequences: societal systems combine two qualities that are commonly referred to as complexity and complicatedness. We address the problem that societal systems remain recalcitrant despite the development of powerful approaches for dealing with both of these qualities. The root of this problem we identify to be that the combination between complexity and complicatedness is emergent; i.e. fundamentally and irreducibly different from either quality in isolation. This means that neither class of such approaches can be expected to work well on their own. But it also means that the obvious strategy of combining theory for complexity and complicatedness may be much more challenging than envisioned. In short, systems where complexity and complicatedness is mixed ought to be treated as a distinct class of systems. Noting a connection to what has long been called "wicked problems" we hereby outline such a class of systems that we call "wicked systems". We introduce a simple model and heuristic and discuss some implications for theorizing and modeling.
\end{abstract}

Keywords:

complexity, complicatedness, wicked problems, wicked systems, models, theory

Preprint submitted to Futures

July 15, 2014 


\section{Introduction}

A mounting scale and frequency of societal and environmental crises has increasingly brought about a realization that we must broaden the range of factors that affect the direction of societal evolution to include societal and environmental values. This means not least that we must break out of the hegemony of the present and near future. But to do so has turned out to be more easily said than done. Societal change and innovation has always been a process that basically unfolds spontaneously, without much overall control and monitoring, and the dynamics of societal systems is also more and more being identified as inherently hard to control and predict. In short, society, and the larger global systems that it is part of, are being identified as complex systems.

All of this clearly changes the landscape for policy (see e.g. Byrne, 2005; Scoones et al., 2007; Leach et al., 2010). The question of how to predict and optimize the future is changing into an acceptance of the futility of such aims and the aiming for other goals such as resilience and sustainability. On the one hand, this raises serious questions about the efficacy of many of the standard policy tools, most of which are designed under entirely different assumptions about how societal systems work. Indeed, it challenges even our basic intuitions about how societies evolve. But, on the other hand, it has also opened up the promise of entirely new types of analytical and policy tools, based on ideas about how we can dynamically steer and scaffold society by engaging more directly with its causal mechanics; for example more bottom-up approaches like the management and design of social networks of actors (e.g. Lane et al., 2011) or the management of innovation dynamics (e.g. Loorbach, 2010).

The application of complexity science is today increasingly suggested as a solution to the new set of challenges that arises from this change in outlook. As we shall see, it is not a trivial matter to delineate complexity science, and it probably cannot be done to universal satisfaction. What we do here is to identify a mainstream of complexity science, with a dominant position from which is has come to set the research agenda, and in effect also thereby define what the concept of complexity means. This mainstream represents a mainly formalist and simulation-based approach to complexity, with its roots in the natural sciences, that has proven highly capable of analyzing many types of complex systems that have otherwise been impenetrable to formal approaches. In the social sciences, the promise is basically the ability to internalize complexity into models of large-scale societal phenomena. The hope is that, like holistic narrative-based approaches, complexity science could deal with historical path-dependent change, but in a more formal and analytically powerful way, and with the crucial addition of providing a theoretical grasp of non-linear dynamics and emergence. It would thereby be able to address a widely recognized need for what we might call a higher theoretical level of resolution (see e.g. Malerba, 2005; Geels, 2010; Holtz, 2011), and be key to a move "from promising to delivering" with regard to this new complex outlook on society.

But despite what looks like an obvious match, and despite a good deal of effort in this direction, the success of complexity science has been mainly limited 
to rather simple social systems, such as crowds, traffic or evacuation, where the complexity of human behavior is reduced to simple rule-following. Success in the study of societal systems in their full complexity has been much more limited. Although concepts like path-dependency, attractors, tipping points and chaos have provided basic lessons that have transformed deeply seated ideas about causality in society, these are highly general lessons that have proven hard to operationalize and they are not strongly represented in policy work. In many - if not most - branches of social science, complexity science remains rarely or at least superficially used. Complexity science appears to remain in a state of being perpetually promising in relation to social science.

We think complexity science is crucial for understanding of how societies work, but that to really make a difference it must be more carefully placed into the context of other basic approaches to understanding systems. We will argue that it is really suited for dealing with only a subset of those systems where we are hampered by the presence of non-linearity and emergence.

Our aim in this paper is to make this problem more explicit and workable, and to contribute an analysis of why complexity science, and indeed formal approaches in general, has proven to be so hard to apply to the social sciences. The paper is structured as follows. First, we introduce the distinction between complexity and complicatedness that is central to our argument, declaring in the process what we mean by "complexity science". Second, we go on to characterize systems that mix these two qualities as "wicked systems" and introduce a model and a heuristic that allows us to chart the relations between problems, systems and methods. Our third point is the question of what wickedness is and why "wickedness is so wicked" - i.e. what is it that makes these systems so hard to address using formal approaches. Fourth, we argue that combining approaches for dealing with complexity and complicatedness separately, which is otherwise a methodological strategy that suggests itself, has worked poorly so far. Using the introduced tools, we offer an analysis of why this would be so problematic. Sixth, and finally, we offer some thoughts about further implications and future directions.

\section{Complex, complicated and wicked}

We begin from a distinction between complexity and complicatedness (also referred to as dynamical and structural complexity; see e.g. Érdi, 2008). These two system qualities are often juxtaposed and contrasted for the purpose of explaining what complexity science is really about. A Google search for "complex vs complicated" yields a wealth of examples, and the nature of these demonstrates that this is a distinction that is perceived of as consequential and of practical relevance. When opposed in this way, complexity is associated with bottom-up self-organization ${ }^{1}$ - like the behavior of a school of fish or a crowd

\footnotetext{
${ }^{1} \mathrm{~A}$ minimal characterization of self-organization, which is a central concept in complexity science, typically includes the emergence of order without any centralized or external descrip-
} 
- while complicatedness is associated with top-down organization, such as in engineering $^{2}$. The distinction is really used for explaining what complexity is, by specifying something that it is not, but that it might otherwise be mistaken for, namely complicatedness. So complexity, then, would roughly be what we intuitively think of as complexity, but minus complicatedness. That may not go a long way as a formal definition of complexity, or even as an intelligent discussion about what complexity can be argued to be, but we think it does say something about the practice of complexity science.

A quick glance at the history of complexity science may tell us something about how this practice and (largely tacit) meaning of the term complexity has emerged. From very early on, the Santa Fe Institute (SFI) came to act as a powerful uniting and aligning force in what can today be referred to as complexity science. The SFI was the first dedicated research center for complexity science, founded in Santa Fe, New Mexico in 1984, by a group of highly influential scientists, many of which were active at the nearby Los Alamos National Laboratory, with roots in the Manhattan Project, and thereby also in the origins of scientific computing and dynamical systems theory in general; see e.g. Galison (1997). Many important ideas about complexity are of course older than, and unrelated to, the SFI and its precedents. Qualitative social science, for instance, often recounts a very different heritage (in systems theory, see e.g. Sawyer, 2005; Vasileiadou and Safarzyska, 2010). But it remains the case that, from the 1980s and onward, the SFI came to define a mainstream of complexity science, and thereby also, in practice, the concept of complexity as understood by scientists, policymakers and the public.

The SFI was created as an explicitly multidisciplinary center, but although it was, and remains, highly multidisciplinary, it is not as methodologically diversified. The methodology that was (and still is) mainly pursued at the SFI is formal, quantitative and closer to natural science and quantitative social science. $^{3}$ At the heart of this methodology, as a critical enabler, lies computer simulation and the capability to describe the entities and interaction rules of a dynamical system and put it all into motion. This is an extremely flexible methodology that made it possible to study and visualize dynamics that used to be utterly inaccessible to the human mind - aided or unaided. Above all, it makes possible a systematic inquiry into emergence in dynamical systems.

Fascination for this new capability and its potentials was a powerful impe-

tion of the order that emerges. In particular, it is microscopic order that, through a dynamics, gets extended to macroscopic scales. Although it has been developed mostly in physics, biology and for social systems, as part of a larger complexity science movement, it originally arose in neither of those fields but in psychology (Ashby, 1947); for more on the history of the concept of self-organization, see Shalizi (2008).

${ }^{2}$ An earlier systems-theory era notion of this sort of complexity is that of interactional complexity (Wimsatt, 1975) which is defined as the degree of cross-coupling in a systems.

${ }^{3}$ In the mission statement of the Santa Fe Institute one can see that the commitment to quantitative approaches is quite explicit: "SFI combines expertise in quantitative theory and model building with a community and infrastructure able to support cutting-edge, distributed and team-based science" (our emphasis). 
tus in the formation of complexity science worldwide with the SFI as a hub. Furthermore, although it predates, and is not necessarily a proper part of it, complexity science is underpinned by a set of fundamental concepts for dealing with non-linear dynamical systems, such as bifurcation, path-dependency and chaos etc, most of which originate from chaos theory (see e.g. Ott, 2002; Cvitanovic et al., 2005) but also from other traditions such as dissipative systems theory (Prigogine and Nicolis, 1977) and synergetics (Haken, 1977).

The typical model in this tradition has a microlevel of agents/nodes existing in a pre-defined environment. What is studied (and subject to empirical testing) are the patterns that arise on an emergent macrolevel from the dynamical interaction between these agents/nodes. Individual traditions and scientists may be more or less strongly aligned with this mainstream, but anyone claiming to work with "complex systems" must relate to it in one way or the other.

So our "definition" of complexity is (i) that complexity as a concept is constructed by the main of the community that works with complexity; (ii) that the concept of complexity thereby reveals the limits of applicability of a particular theoretical toolbox. So we think that it points to (iii) a specific class of systems that happens to be amenable to analysis using that particular toolbox ${ }^{4}$. What is key to our differentiation between complexity and complicatedness is this: although complexity and complicatedness are clearly linked in numerous ways, they still present us with two radically different sets of methodological and theoretical challenges.

So what about societal systems in this mix? Are they complex or complicated? On the one hand, societies are undeniably complicated with their multi-level organization and bewildering array of qualitatively different and interacting entities. Systems theories for example seize upon what is seen as an irreducible complicatedness of societal systems. Yet society is also often, and quite convincingly, argued to be a complex system in the bottom-up selforganization sense (e.g. Sawyer, 2005; Castellani and Hafferty, 2009; Ball, 2012), and it can certainly be argued that much of its complicated structure arises from bottom-up rather than top-down processes. We clearly see no reason why systems could not be both complicated and complex at the same time, and societies would appear to be an excellent example of such a type of system. Systems that combine these two qualities will here be referred to as "wicked systems". We will soon go into the details of what that means, but for now we will just use the term as a label.

It is precisely for those "wicked systems" that we claim that scientific practice and expectations become confused, and where we think that the compass for future scientific development has been lost. Let us illustrate this by considering some different viewpoints on society and complexity, all representing insights,

\footnotetext{
${ }^{4}$ See also Byrne's (2005) concept of "simple complexity", and Morin's (2007) concept of "restricted complexity", which are in line with how we conceive of this complexity science mainstream; also Byrne and Callaghan (2014) who, from the standpoint of Morin's terminology, discuss the dominance of this mainstream.
} 
but insights that we think are hampered by the lingering confusion that we here aim to address.

For example, when Ball (2012) speaks of society as a complex system, he appears to mean complexity in a wide sense that encompasses both complexity and complicatedness. But the models that are covered really deal with complexity only in the more narrow sense used here. We may only presume that he thinks that complexity of the structural sort (i.e. complicatedness) should be explained by models working on the basis of dynamical complexity (i.e. complexity in the here-used dichotomy). This is a micro-macro view, quite typical of positivist science, and of mainstream complexity science, where micro is seen as prior to macro, and thus the universally proper locus of explanation.

Walby (2007) provides a lucid analysis of what complexity science (or, theory, in her parlance) could and should contribute to sociology, and her picture of societal systems is clearly one of what we refer to as "wicked systems"; i.e. combined complexity and complicatedness. But in her analysis of how this contribution should occur, where she applies the concepts of complexity science, she ends up where others have frequently ended up: in a tantalizing but unfulfilled promise of something more and deeper; in a perpetual feeling that we are close to a breakthrough and a paradigm shift. In their native domain, the complexity concepts that she applies are crisp, well defined and methodologically powerful. When applied to systems that are both complex and complicated they still make a lot of sense, but they also somehow seem to lose their theoretical bite.

The distinction that we propose between complex and wicked systems can also be interpreted in the context of the sociological discussion about the meaning of complexity in societal systems by e.g. Byrne (2011, 2013); Byrne and Callaghan (2014). Their view of societal complexity is referred to as "complex realism", which can be described in terms of sociological traditions as critical realism informed by complexity science (Reed and Harvey, 1992; Harvey and Reed, 1996). Using the terminology of Morin (2007), these authors differentiate between two perspectives on complexity as "restricted complexity" and "general complexity", where the latter is similar to our description of wicked systems, and the former to what we here refer to as mainstream complexity. However, the separation between general and restricted complexity is treated as simply two separate perspectives on the same thing, namely "complexity". The point that we wish to make is that these two perspectives are better understood as descriptions of different things: general complexity does not actually describe complexity, but rather wickedness. We understand complexity as part of wickedness, but we do not thereby see wickedness as a type of complexity. This can perhaps be likened to how the fact that the color red is a component color of yellow does not mean that we can describe yellow as a type of red. Our point therefore fundamentally transforms the meaning of this discussion: general complexity is in our view not a generalization of restricted complexity, it is a description of something of which restricted complexity is a component, but that is qualitatively different than restricted complexity.

Surprisingly perhaps, the possibility of systematically exploring the consequences of mixing complexity and complicatedness appears not to have been ex- 
plicitly pursued ${ }^{5}$. As things stand, the mainstream of complexity science may be aware that complexity and complicatedness are distinct qualities, but complicatedness in complex systems is not seen as a fundamental problem. Complicated or not, they are complex, and that is what is seen as fundamentally important: extending mainstream theory to deal with them is seen as challenging and hard, but, essentially, gradual and cumulative work. Sociological complexity thinkers disagree with mainstream complexity science about how complexity ought to be understood. Notably, however, they do not really disagree that, in the end, complexity ought to be one single thing. Our analysis will lead us to a different conclusion. We do not see societal systems as a type of complex systems, but as a type of system where complexity is mixed with complicatedness, yielding an emergent quality - wickedness - to which neither complexity science, systems approaches, mathematical models or combinations between them lend themselves very well.

\section{Understanding the co-evolution of methods, problems and systems}

We will now introduce and explore a simple model and heuristic aimed at helping us to better understand relations between methods, problems and systems. We focus on the question of why mainstream complexity science would be so hard to extend to "wicked systems", but we think this question is more general than that. We think it boils down to why formal science in general would be incapable of dealing with these systems.

In Figs. (1-4) we chart out system types, problems and theoretical approaches on the basis of these two system qualities. The corners of the plane that is described give us four ideal system types. Systems that are neither complex nor complicated (bottom-left corner) we call "simple systems", systems that are complex but not very complicated we call "complex systems", systems that are complicated but not very complex are labeled "complicated systems" and, finally, systems that are both complicated and complex we call "wicked systems". We may view these figures as either four-field graphs or graphs with continuous axes, corresponding to whether we prefer to see complexity and complicatedness primarily as binary qualities or graded quantities.

We choose the term "wicked systems" in recognition of a potentially deep connection (whose exact nature remains to be worked out) between this class of systems and what has been called "wicked problems". The term "wicked problems" was first coined in management research by Horst Rittel (briefly introduced by West Churchman, 1967) to characterize a class of problems that failed to fit into the molds of the formal systems theoretical models that were being applied across the board at the time with considerable confidence. Just about any large-scale societal problem can in fact be confidently put into the

\footnotetext{
${ }^{5}$ At least not recently and with the benefit of a more developed science of complexity at hand. But see Wimsatt (1975) for an early and interesting analysis that clearly goes a long way in this direction; also re-printed in Wimsatt (2007).
} 
category of wicked problems: starvation, climate change, geopolitical conflicts, social disenfranchisement, and so on. All these are problems that escape definition and where there is a constant feeling that the efficacy of proposed solutions is called into question not only with regard to feasibility and adequacy but also with regard to the risk of creating cascades of other problems that are impossible to foresee and that may be even worse than the initial problem (see also Leach et al., 2007; Scoones et al., 2007). Explicating the concept, Rittel and Webber (1973) conclude that the domain of wicked problems in social systems is vast it includes just about any problem short of trivialities. In Churchman's (1967) words, what we do with wicked problems is to either tame them by creating "an aura of good feeling and consensus" or by "carving off a piece of the problem and finding a rational and feasible solution to this piece"; this would appear to well describe also our generalization of "wickedness". By considering "wickedness" as a system quality, we generalize to also be able to speak of things like wicked dynamics, wicked phenomena and wicked systems.

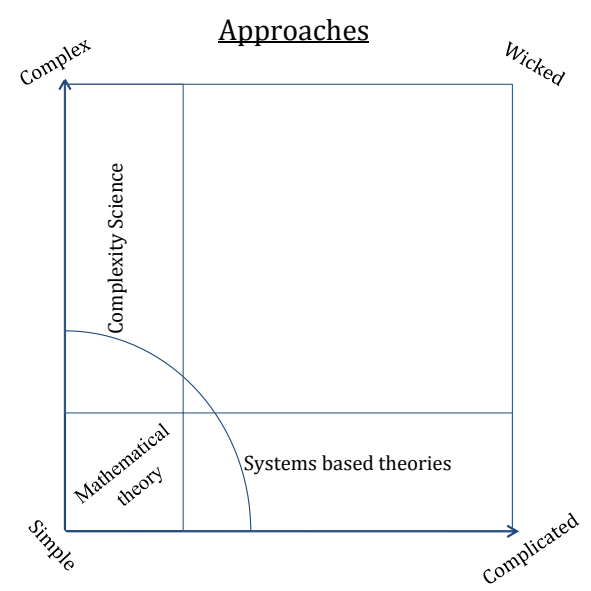

Figure 1: On a plane described by a complexity- and a complicatedness axis, we here may schematically indicate what sort of problems that different approaches are strong at dealing with.

In Fig. (1) we map three groups of formal theoretical approaches into the complexity-complicatedness plane: "mathematical theory", "systems based theories" and "complexity science". Under the rubric of "mathematical theory" we place theory and models mainly based on closed-form equations (whose scope have later been expanded with numerical methods), most importantly in this context neoclassical economic theory, such as mainstream macroeconomics approaches, which are characterized by strong assumptions about agent rationality and equilibrium that serve to make models mathematically tractable. When we refer to systems based theories we mean approaches that rely on holistic ontologies; systems theory is a central example of such approaches although the 


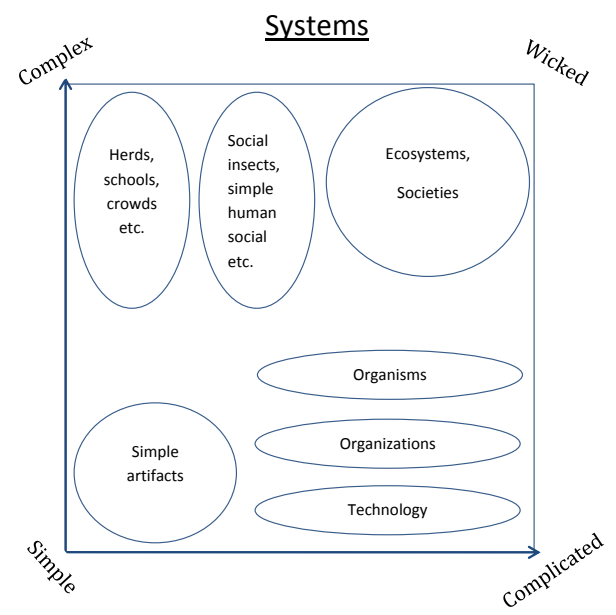

Figure 2: We here map different types of systems onto the complexity-complicatedness plane to roughly indicate where many or most of the problems encountered in different types of systems fall. Near the "complex" and "complicated" corners we find paradigmatic examples, such as schools of fish and technological artifacts, respectively. In the "wicked" corner we find examples, such as ecosystems and societies, that are probably straightforward to accept as partaking in both complexity and complicatedness at the same time. As we move away from the corners, the placement of the examples in the diagram becomes more contentious and it is well to point out that the idea here is not to precisely classify the systems that we have used as examples. There is considerable room for argument about where they could be placed, how they should extend across the diagram and what exceptions that may exist. We view it as a strength of the diagram that it can serve as a basis for such discussions. By "simple human social" we mean social sub-systems that are relatively unstratified in their organization and that, although part of a greater societal system, may be considered to some extent in isolation and in terms of agents and environments on a single level of organization; say for instance the social interactions between children in a schoolyard. These would be examples of social systems that can be straightforwardly studied using agent-based simulations. 


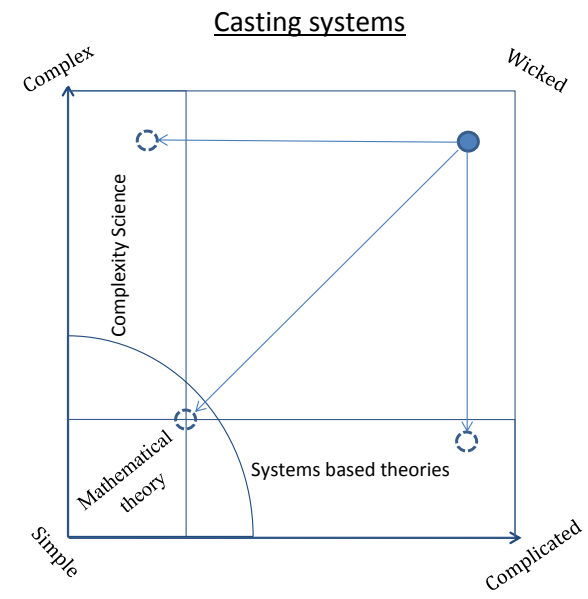

Figure 3: We here illustrate the casting of wicked problems as either complex, simple or complicated problems so as to enter them into formal machineries.

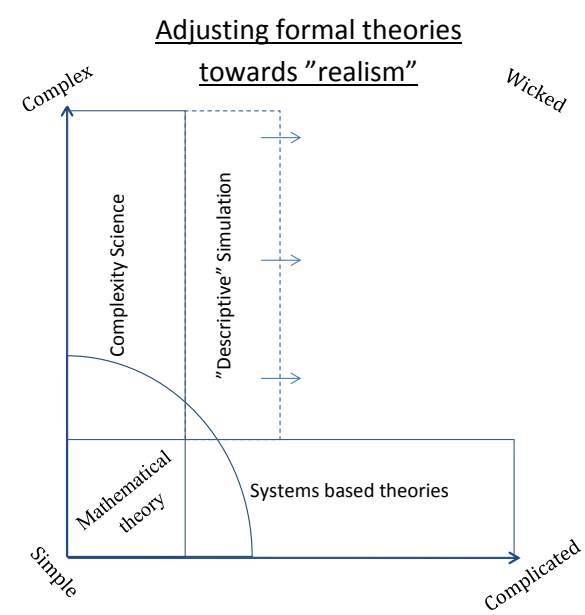

Figure 4: Here we illustrate how descriptive, or agent-based, simulation represents an attempt to expand complexity science from the complex corner towards the wicked corner. This demands models that are more complicated and that will share features of what we call systems theories. 
category is wider than that. What can be more generally termed "systems thinking" (e.g. Weinberg, 2001), for example, permeates science exceptionally widely today. Systems based theories break with the reductionist tradition of mathematical theory by making the structure of societal systems explicit on a larger scale, deeming them to be irreducible: this has expanded the reach of formal methods from the simple corner towards the complicated corner, generally following the example of engineering. Finally, complexity science has revolutionized science on a fundamental level by covering an important flank that used to be so hard to deal with that it was nearly entirely unexplored before the 1980s. It mainly expands from mathematical theory, but also to an important extent from systems based theories with which its shares a strong attention to feedback processes. As we illustrate in Fig. (4), agent-based simulation, or more generally "descriptive simulation" (see Edmonds and Moss, 2005), expands from the simple-and-complex towards the complicated-and-complex side, and in doing so it comes to share features with systems based approaches; more on this later in the paper.

Societal systems would have their center of gravity near the wicked corner of the graph (see Fig. 2), but since formal approaches are unable to access many of the problems that societal systems present us with, they will selectively address sub-problems that happen to fall into their domains or transplant problems from near the wicked corner to the corners of their preference (see Fig. 3) ${ }^{6}{ }^{\text {In }}$ the former case we obtain important but limited "snapshots" of the system in question and we are, as we will discuss later, faced with the problem of how to combine such snapshots; see also Wimsatt (1975). In the latter case we may get spurious results where strong assumptions mean that the benefit of accessing formal methods of analysis does not warrant the price in realism.

Most complexity scientists will readily admit that their influence is concentrated to the region near the complex corner. But since complexity science defines itself more generally as dealing with systems of high complexity (falling toward the top of the complexity axis) there is no systematic recognition that something about complex systems may change qualitatively along the complicatedness axis. In summary, it is easy to identify powerful scientific approaches for dealing with all parts of the plane except for the wicked corner.

To begin understanding what a mix between complexity and complicatedness entails, we should first note that we are mixing a primarily structural quality complicatedness - with a primarily dynamical quality - complexity - and that both of these on their own are theoretically challenging. To make matters even worse, the way in which they intermix cause these qualities to fuse into something quite unlike either quality in isolation. This is what we mean when we say

\footnotetext{
${ }^{6}$ Organisms may seem strange bedfellows with machines near the complicated corner. But although organisms are generally closer to the Wicked corner than machines they are in fact quite strictly organized. Just like machines they generally do not change during their lifetime: they have an assembly/ontogenetic phase followed by another phase during which they have a nominal ecological function (in some instances this pattern will recur, such as in metamorphosing species like dragonflies, but this happens in a highly pre-ordained way.)
} 
that the combined quality of "wickedness" is emergent. Complexity and complicatedness can be seen as mutually reinforcing in societies and ecosystems our two principal examples of wicked systems. Self-organization here generates, changes and maintains macro structure, and macro structure, in turn, scaffolds and creates a multitude of arenas for self-organization. We will now delve at least somewhat deeper into to this observation in order to understand better how and why wicked systems come to mix complexity and complicatedness: Where do they come from? How are they maintained? What sets them apart from complex and complicated systems?

\section{Wicked Systems as poorly decomposable systems}

The constituents of wicked systems constantly try to outsmart one-another, reaping their own benefits, reacting to threats from other constituents. They constantly enter into new constellations, dissolve old constellations, and react to the immediate situation around them. What we get is a situation where complicated organization and complex dynamics is in a constant state of re-negotiation, constantly challenging any settlement of the system into a level hierarchy, constantly facing the system with qualitative novelty that other components have to react to. They are more like arenas for interaction between entities (typically complicated systems like organisms and artifacts) than they are like systems with overall top-level functions and agendas of their own. When we imagine a desirable society, we do tend to imagine it as a good arena for interaction, yielding equitable and sustainable patterns of interactions for its denizens.

Such systems, as we know, can be tremendously persistent despite all this upheaval. In fact, since they lack an overall function (at least in a straightforward sense, see e.g. Schlosser, 1998), the very concept of them breaking down is not straightforward. A breakdown of societal or ecological systems typically does not mean that they cease to operate (like a car that broke down and sits at the side of the road), but that the conditions for (and makeup of) the components change in a dramatic way; species going extinct, humans starving, being oppressed and so on. We may expect them to be persistent in the sense of having continuity, but less persistent in the sense of providing an arena for interaction that we see as desirable.

It is not hard to imagine why systems of this description would be challenging to understand formally - but can it be understood formally why they cannot be understood formally? The benefit of achieving such an understanding could be substantial as it could offer a more detailed map of what, more precisely, the methodological problem with these systems consists in. Such an understanding, if it can inform us about what approaches are likely to work in which contexts, can also serve to make it easier for formalists and non-formalists to come out of their trenches and collaborate. We will now propose one way of understanding why wicked systems are so recalcitrant in a way that is both formal and intuitive and that should be accessible to formalists and non-formalists alike: the organization and dynamics of wicked systems make them poorly accessible to 
approaches that rely on what Simon (1996) called near-decomposability, which is to say just about any conceivable formal theorizing. ${ }^{7}$

Like any formal scientific approach, complexity science and systems theories strive to isolate systems for independent study such that model properties can be fixed and formally defined. Ceteris paribus, formal models are desirable since they promote clarity and may be amenable to powerful analytical tools based on mathematics and computation. As Byrne (2005) states, the problem with formalist science is by no means that it does not work, it is "... that it works where it works and it does not work everywhere". But what really makes a system suitable for such an approach? Under what circumstances, more precisely, will formal methods run into trouble?

Simon (1996) introduced the concept of "near-decomposability" to explain in a clear and systematic way what conditions that need apply for a system to be possible to study in a formal and controlled manner. The central observation is that if a system is to be possible to study in isolation, its dynamics cannot be importantly disturbed by outside influences. In Simon's parlance we should be able to identify an internal environment where the dynamics that we study takes place, and an external environment that can be assumed to be static, or at least to be variable only in highly regular ways. The boundary between the internal and external environment is the delimitation of the model, and it is referred to as the interface. What we study with a model is then an internal environment. Hierarchical system organization is important here: our internal environment constitutes the external environment of the objects that populate it, and that we deal with only via their interfaces. We deal, therefore, with objects only in the form of interfaces, for example their interactions with other objects in the studied system. The beauty of all this is that it makes the world manageable: we declare our system as autonomous from external disturbance and we hide any complexity and complicatedness residing on lower levels of the hierarchical organization.

We may study this internal environment during what Simon refers to as the short run: a time scale that (i) is long enough that our objects' interfaces are meaningful ${ }^{8}$ and for important dynamics to have time to happen and (ii) short enough that our assumptions about the interfaces remain valid. The greater the separation of scales between the internal and the external environment, the greater will the difference in size and speed of the dynamics on these two levels be, and the more generous will the short run be; i.e. the more interesting things will have time to happen. For example, models of particle physics can gainfully be formulated in this way because those systems exhibit a clear and clean scale separation. Engineered systems, as Simon (1996) points out, are designed to fit into above description.

\footnotetext{
${ }^{7}$ Using Simon's model also has the added benefit that it has been highly influential in shaping both practice and conceptual work in systems and complex systems theory.

${ }^{8} \mathrm{~A}$ human can for instance make decisions (a typical interface feature) over a time scale of minutes, but hardly on a time scale of milliseconds.
} 
Importantly, this scheme can, potentially ad infinitum, be nested hierarchically. At each new level we can reduce both complexity and complicatedness back down to manageable levels again, and this is what allows us to construct systems that, taken as wholes, are mindbogglingly complicated; most notably in engineering, but they same principle applies to biological organisms. The parts of such a system can be improved independently, with respect to identifiable functions, as long as those functions in the system are retained. For example, it is straightforward to replace the engine of a car with another engine with the same function, but, say, an improved fuel economy. In fact you can do anything to a component as long as you do not alter its interface. Significantly, the components of technological artifacts and organisms also have no separate agendas; it makes no sense for the engine of a car to benefit on the expense of the car as a whole since engines are meaningless objects except as part of a functional whole. Multicellular life, with cell types, tissues and organs, is no different in this respect. Selection for top-level functionality often yields hierarchical systems with near-decomposable levels (such as engineered artifacts or organisms) since such an organization increases evolutionary adaptability (Wimsatt, 1975; Simon, 1996).

In many important cases we can surely make assumptions of near-decomposability for societal systems, and when we can we are able to bring powerful scientific approaches to bear. For the purposes of complexity science it would seem reasonable that certain subsystems, such as traffic or crowd behavior, can be argued to fit this description. The dynamics of cars and people play themselves out over much shorter time scales than that on which urban systems, roads, traffic regulation and so on, change. Such phenomena are also often ephemeral, which bounds the problem even further. For example, at night the traffic jam dissipates and leaves no traces that affect tomorrow's traffic. Similarly may be argued for certain highly abstractly conceived phenomena that depend on persistent features such as network dynamics, geography, basic resource constraints, strategic dilemmas and so on.

But what about societal phenomena more in general? For example, what about sociotechnical transitions or other wicked problems? Sociotechnical systems in general are open systems, in which many and far-flung social, technical and natural processes co-exist, co-evolve and have an impact on each other on overlapping timescales and levels of organization. In many cases these problems unfold across time scales of decades or more. They involve discontinuous, qualitative change as well as cascade effects (e.g. Lane, 2011) whereby change strongly and rapidly feeds back into the conditions for further change. Such systems are, to say the least, hard to contain in a Simonean compartment with a "short run" over which, for example, transitions can be studied against the background of an unchanging external environment. The fundamental problem for complexity science in this context, and really any approach that relies on these ontological assumptions, is that on the time scales of sociotechnical change almost everything in society really is "changing with everything else" (Malerba, 2005): there is no relevant "short run" for a model to operate in; there is no way of cutting the system into distinct and persistent levels of organization. 


\section{The obvious way forward and why we keep bogging down}

We may crudely summarize the formal theoretical situation as follows. Macroeconomic models drag society towards the simple corner of the plane illustrated in Figs. (1-4), which brings it under the sway of analytical or numerical mathematical methods. This makes for supremely formal and powerful models, but it often comes at the cost of moving societal problems far from their "empirical home". Systems theoretical approaches emphasize the complicatedness of society and attempt to understand, design and steer it as a complicated but not complex system, approximately in analogy with machines and organisms. By doing this, society is moved from near its native wicked corner to near the complicated corner. Although systems theories catch more of the structure and dynamics of society than macroeconomic theory does, they again bring society out of its right element in order to subject it to certain tools of analysis and design.

The call of complexity science is that society is neither simple nor complicated - it is a complex system - so we should use these new complexity methods for understanding it (see e.g. Epstein and Axtell, 1996; Epstein, 2007; Squazzoni, 2008; Ball, 2012, among many). In evidence that this is a promising track, successes in understanding certain simple and well delimitable social systems, that happen to fall closer to the complex corner, are brought forth. These are often viewed as "steps along the way"; a way that is envisioned as perilous but negotiable using the established methodology, either by linking the formal models or by scaling them up. But by emphasizing complexity in this way, we think that society is again frequently moved from its wicked corner. This time toward the complex corner and, although in a different direction, again out of its right element.

If society is both complicated and complex, it would appear particularly reasonable to use a combination between approaches that successfully deal with complicated and complex systems to provide a more integrated view. For example to combine systems theoretical methods with a complexity approach. This is also in practice what many are attempting to do. Multi-agent simulation (e.g. Gilbert and Troitzsch, 2005), which is basically an extension of simple complexity modeling (such as in physics and chemistry), could for instance be described as such an approach: agents, interaction modalities and environments are designed a priori in a fashion that recalls a systems approach, but they are subsequently let loose in a dynamics where emergent patterns arise, so they are clearly also complexity models. One can also imagine multi-agent and other models being combined in the form of modules in a more explicit hierarchical systems ontology along the lines proposed by Ostrom (2007). This is also an approach typical to Integrated Assessment Modeling (Rotmans and Asselt, 2003) where systems of models are constructed to deal with problems involving large-scale, often global, dynamics; societal sub-systems are usually represented simplistically using general equilibrium economics models. Agent-based models have frequently been used as modules in such models for some time (see e.g. Pahl-Wostl, 2002). 
But are such simple combinations, where models organize complexity into a complicated structure, necessarily suitable for providing a fuller picture of these wicked systems? We would say that they are not. In wicked systems, the rules and entities are not only hard to uncover, they change as a result of the dynamics itself. There are no neat scale separations in wicked systems that allow us to separate ontology from state in the general case. This is what Lane and Maxfield (2005) refer to as "ontological uncertainty" and it is also the reason why wicked systems are "worse than complex".

Another reason why combination works poorly is that the formal methods that we thereby combine derive their power not from the presence, but from the lack of, complexity or complicatedness in systems. They rely on simplifications of either the structure or the dynamics of systems (or both), and for wicked systems we can frequently do neither while maintaining acceptable levels of realism. For example, engineering does not work because technological systems are complicated. It works because technology can be constructed such that it is not complex. For complexity science, even a cursory review of the literature clearly reveals that it excels specifically in dealing with systems toward the topleft corner of this plot; i.e. systems that are complex but not very complicated. Combined approaches thereby combine the weaknesses rather than the strengths of the constituent approaches.

\section{Discussion}

Can this theoretical lacuna near the wicked corner be filled - just like the area around the complex corner is being filled by complexity science? We think that a great challenge for the future lies precisely in understanding wicked systems. In this paper we have focused on the preliminary task of establishing this category of wicked systems, and as a result there has been little space to pursue all the implications that we see as important. Some of these, such as what the implications of our argument would be on the structure-agency debate, would demand an entire article on its own for a satisfying treatment. However, the concept of wicked systems clearly has implications for this discussion concerning the analytic connection or co-relation between individual and social processes, since the conditions under which we see scale separation, and the effect that scale separation has on the efficacy of different approaches, are central to our argument. On the basis of the foundation that we lay here, the exploration of these issues will have to wait for coming papers, as will indeed a more thorough interpretation of the implications for constructing and imagining futures.

In Sec. (5) we concluded that the obvious strategy that (explicitly or implicitly) mixes approaches for dealing complexity and complicatedness in formal models faces serious problems. The reason for this is that, as we argued in Sec. (4), formality fundamentally rests upon an assumption of non-wickedness. We think this contributes to an explanation of why narrative ${ }^{9} /$ qualitative ap- $^{-}$

${ }^{9}$ Narrative is of course the oldest form of theorizing about social systems. There is quite a 
proaches have been so hard to move away from despite vigorous efforts to do so. Arguments to this effect have so far typically been put forward by qualitative scientists in the language of, and for a receptive audience of, qualitative scientists. What we have attempted here could be described as a formal, yet intuitive, critique of formal approaches to wicked systems. Our choice to argue our point in the framework of Simon (1996) should be understood on this background. It was not only suitable for the purpose. Being based, as it is, on robust and time-tested systems thinking, we also think it has a chance of making sense to both quantitative and qualitative scientists.

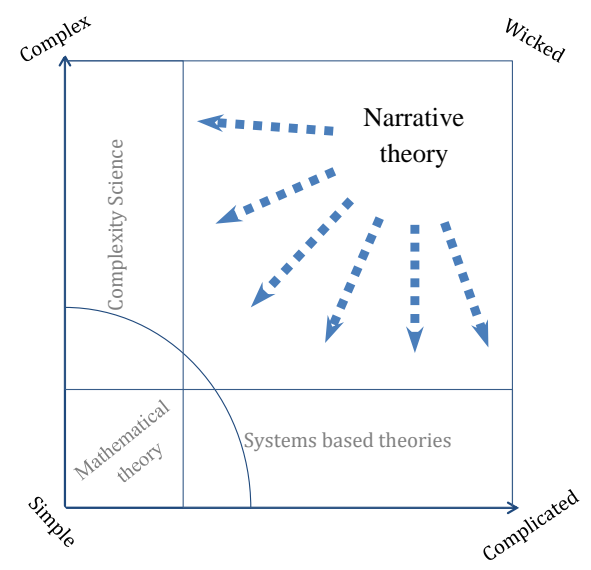

Figure 5: We here map narrative/qualitative approaches together with the formal approaches that we mapped in Fig. (1).

In Fig (5) we add also narrative approaches to the mix, reflecting our observation that the use of narrative in science (and otherwise) is strongly correlated precisely with wickedness. The advantage of narrative is that, unlike formal approaches, it can at least begin to operate in the wicked zone. It can handle a number of key characteristics of wicked systems such as their heterogeneity, their contingency and their multilevel nature. We generally concur with the analysis of Byrne and Callaghan (2014) and Ragin (2009) that narrative, and in particular historical case study, is important for understanding what we mean by wicked systems. The future, of course, is always attached to a future history, and an imagined history has all the characteristics of a remembered history. Narrative in the role of constructing what we might call historical case studies

deal of literature on the concept of "narrative" and how it is important for not only "wrapping our minds around" society, but also to deal with its constant state of change from innovation, re-interpretations and the potential for taking different perspectives on the same thing. There is no room here for a review of this literature, so we point the interested reader to e.g. Geels and Schot (2010) and Lane and Maxfield (2005) and further references therein. 
of the future has also been discussed in the context of future studies for similar reasons (see e.g. Miller, 2007). In our conceptualization, another way of specifying what the strength of narrative is with respect to wicked systems, is that it is the only mode of theorizing that is not obviously married to assumptions of a lack of either complicatedness or complexity; which, as we will soon get back to, does not mean that it is particularly good at dealing with their presence.

Beyond its use in theorizing, which goes back all the way to the roots of philosophy, the suitability of narrative for understanding matters concerning social life is hardly a happy coincidence. It is linked to our cognitive apparatus and as such a result of evolutionary cognitive adaptation to a multi-million year history of living in communities that are simultaneously complex and complicated; see e.g. the so-called "Machiavellian intelligence hypothesis" (e.g. de Waal, 1982; Byrne and Whiten, 1989; Whiten and Byrne, 1997; Whiten and van Schaik, 2007).

But this also points to the limitations of narrative and the necessity of formal approaches. In particular in the context of modern societal systems, our abilities (while impressive on a zoological comparison) may be quite insufficient for our purposes. Our ability to handle complicatedness is quite limited, not least by a limited short-term working memory (see e.g. Coolidge and Wynn, 2005, 2006; Read, 2008), and our ability to make strict inferences and abstractions without the use of mathematics and formal logic is poor. When it comes to our ability to handle complexity, it is worse than poor: it is treacherous.

Consider for instance Schelling's (1978) tipping model as an example. The arguably most remarkable contribution of this model is that it formulates both a surprising question and a surprising answer at the same time. The model basically shows that even tiny preferences about what neighbors that we would like to live among can quickly lead to complete segregation. The whole dynamics thereby turned out to work in a way that is qualitatively different than what our unaided intuition would tell us. Non-linearity and emergence are treacherous because they often throw us off without us even noticing it. We think that we understand the problem. We may even think that the solution to the problem is so obvious that we need not even bother to consider it very closely.

To tackle complexity head-on we absolutely need formal models, and in particular simulation models since they provide a flexibility that we cannot do without. The cognitive engine that runs the narrative must be complemented with formal theorizing to get past impasses where reliance on intuition will get us no further, and complexity is perhaps the steepest of those impasses.

The reader may here interject that narrative theorizing is used in conjunction with formal models already. This is certainly true. The problem is that a clear and formulated understanding of (i) why it would be a good idea to do so, and (ii), how it ought to be done, is notably lacking. In particular we are lacking an understanding in a format that would be acceptable to at least a notable fraction of those close to the formalist mainstream of complexity science. 
Under the formalist and reductionist ${ }^{10}$ ideals that govern the mainstream of complexity science, narrative based strategies clearly remain on the fringe. They are maximally tolerated as something that we are constrained to doing "in the meanwhile", before we succeed in coming up with formal models. Many indeed see narrative approaches as precisely what complexity science should aid us in "moving past". We hope to have contributed to a better foundation for questioning, in a systematic way, that sentiment of a priori rejection of qualitative approaches.

As indicated in Sec. (2), the system quality that we here call wickedness has been described under other labels in the literature, and typically as a type of complexity. For example, Byrne (2005) speaks of "complex complexity" as opposed to "simple complexity", and Stewart (2001) talks about "incondensable complexity" as opposed to "simplicity". In both these cases the former concept clearly concern what we call wickedness. Byrne and Callaghan (2014) and Morin (2007) use the term "general complexity" in opposition to "restricted complexity" where the former, however, contains, rather than is complementary to, the latter. It would thereby correspond to the entire top region of our complexity-complicatedness plane (see Figs. 1-4). The set of problems that we associate with wickedness, and the confusion that surrounds them, also come to the fore clearly in the literature about hierarchies and organizational levels. See for example the analysis by Lane (2006) of, on the one hand, the partly overlapping and partly contradictory views on the relation between hierarchy and complexity by Holland (1998), Anderson (1972) and Simon (1996), and, on the other hand, views about whether the concept of hierarchical levels is useful at all in social science by Latour (1993) and Ginzburg (1989).

To summarize our contribution in this context, we have identified wickedness as a distinct quality that is related to both complexity and complicatedness, but as an emergent combination between these qualities, rather than as a type of either quality. We illustrated this in Sec. (2) metaphorically by stating that red is a component color of yellow, but yellow is not thereby a type of red. Using the introduced diagram we have been able to place systems, approaches and problems in to the context of each other, facilitating a better understanding of their interrelations and, not least, of why systems near the wicked corner of the diagram are so hard to understand. We think that one major benefit of our conceptualization here is that it removes the otherwise so strong prior notion that societal systems should be understandable by extending the toolbox of mainstream complexity science. Having identified that complexity science has mainly evolved to deal with a related, yet distinctly different, type of systems,

\footnotetext{
${ }^{10}$ Practitioners of mainstream complexity science would take strong issue with being thus labeled, and to a large extent it is all a matter of what we compare with. If we compare with natural science and quantitative social science, they do take issue with fundamental reductionist assumptions by the very fact that they accept and systematically study emergence. On comparison with more qualitative approaches, however, their focus on studying emergence from the perspective of basic organizational levels means that they tend to disregard multi-level organization and still come across as reductionist.
} 
we confirm the intuition that complexity science has an important part to play, but we have also redefined its role and invited other approaches in alongside with it. We have not offered much on the issue of how to combine such approaches, but considering the vast scope of that question, we think that setting the stage even slightly better for addressing it is an important step forward.

By shifting the picture of society and complexity in this way we hope to have opened up new avenues of thinking on this important issue, which is central not only to future studies, but in any context where the evolution of wicked systems is considered. The theoretical challenges that we discuss here are by no means unique to the social sciences, and that we may be well served by looking to developments in other fields for inspiration, as a guide and as a source of models. Most importantly, evolutionary biology, has over the past few decades undergone a dramatic transformation. The new theoretical landscape that has emerged (sometimes broadly referred to as an "Extended Synthesis", referring back to the "Modern Synthesis" and the formation of neo-Darwinism; see e.g. Pigliucci and Müller, 2010) has invigorated a class of biological theories ${ }^{11}$ that maintain a grounding in quantitative theorizing but that also has deep similarities with qualitative theories in the social sciences. In the context of our thesis, what is particularly interesting is that they have clearly begun tackling what we call wickedness, and not least that it addresses the question of how such systems evolve and how they are generated and maintained. This opens up an interesting prospect for exchange between the biological and social sciences on a different level than what has formerly been the case; see for example the application of such theories to cultural evolution by Andersson et al. (2014).

\section{Acknowledgements}

We wish to acknowledge the support of the EU-FET grants MD (no. 284625) and INSITE (no. 271574). The paper has benefitted from discussions with David Lane, Björn Sandén, Duncan Kushnir and several others at our department.

Anderson, P. W., 1972. More Is Different. Science 177 (4047), 393-396.

Andersson, C., Törnberg, A., Törnberg, P., Apr. 2014. An Evolutionary Developmental Approach to Cultural Evolution. Current Anthropology 55 (2), $154-174$.

Ashby, W. R., 1947. Priciples of the Self-Organizing Dynamical System. Journal of General Psychology 37, 125-128.

Ball, P., 2012. Why Society is a Complex Matter: Meeting Twenty-first Century Challenges with a New Kind of Science. Springer Verlag, Berlin, Heidelberg.

\footnotetext{
${ }^{11}$ E.g. Developmental Systems Theory (e.g. Oyama et al., 2001), Generative Entrenchment (e.g. Wimsatt and Griesemer, 2007) and Niche Construction (e.g. Odling-Smee et al., 2003)
} 
Byrne, D., Oct. 2005. Complexity, Configurations and Cases. Theory, Culture \& Society 22 (5), 95-111.

Byrne, D., 2011. What is an effect? Coming at Causality Backwards. In: Williams, M., Vogt, P. W. (Eds.), The SAGE handbook of Innovation in Social Research Methods. Sage Publications, Ch. 4, pp. 80-94.

Byrne, D., Jul. 2013. Evaluating complex social interventions in a complex world. Evaluation 19 (3), 217-228.

Byrne, D., Callaghan, G., 2014. Complexity Theory and the Social Sciences: The State of the Art. Routledge.

Byrne, R., Whiten, A., 1989. Machiavellian Intelligence : Social Expertise and the Evolution of Intellect in Monkeys, Apes, and Humans. Clarendon Press, Oxford.

Castellani, B., Hafferty, F. W., 2009. Sociology and Complexity Science: A New Field of Inquiry. Springer Verlag, Berlin, Heidelberg.

Coolidge, F. L., Wynn, T., 2005. Working memory, its executive functions, and the emergence of modern thinking. Cambridge Archaeology Journal 50, 230-231.

Coolidge, F. L., Wynn, T., 2006. The effect of enhanced working memory on language. Journal of Human Evolution 50, 230-231.

Cvitanovic, P., Artuso, R., Mainieri, R., Tanner, G., Vattay, G., 2005. Chaos: Classical and Quantum. Niels Bohr Institute, Copenhagen.

de Waal, F., 1982. Chimpanzee Politics: Power and Sex Among Apes. Jonathan Cape, London.

Edmonds, B., Moss, S., 2005. From KISS to KIDSan 'anti-simplistic' modelling approach. Multi-Agent and Multi-Agent-Based Simulation, 130-144.

Epstein, J. M., 2007. Generative Social Science: Studies in Agent-Based Computational Modeling (Princeton Studies in Complexity). Princeton University Press.

Epstein, J. M., Axtell, R. L., 1996. Growing Artificial Societies: Social Science from the Bottom Up. The MIT Press.

Érdi, P., 2008. Complexity Explained. Springer Verlag, Berlin, Heidelberg.

Galison, P., 1997. Image and Logic: A Material Culture of Microphysics. Univerity of Chicago Press.

Geels, F. W., May 2010. Ontologies, socio-technical transitions (to sustainability), and the multi-level perspective. Research Policy 39 (4), 495-510. 
Geels, F. W., Schot, J., 2010. Process Theory, Causality and Narrative Explanation. In: Grin, J., Rotmans, J., Schot, J., Geels, F., Loorbach, D. (Eds.), Transitions to Sustainable Development. Routledge, New York, Ch. I.6, pp. 93-101.

Gilbert, N., Troitzsch, K. G., 2005. Simulation for the social scientist. McGraw Hill International.

Ginzburg, C., 1989. Clues, myths, and the historical method. John Hopkins University Press, Baltimore, MD.

Haken, H., 1977. Synergetics - an introduction; Nonequilibrium Phase Transitions and Self-Organization in Physics, Chemistry and Biology. SpringerVerlag.

Harvey, D. L., Reed, M., 1996. Social science as the study of complex systems. Chaos theory in the social sciences: Foundations and applications, 295-323.

Holland, J. H., 1998. Emergence. : Addison-Wesley, Redwood City, California.

Holtz, G., Dec. 2011. Modelling transitions: An appraisal of experiences and suggestions for research. Environmental Innovation and Societal Transitions 1 (2), 167-186.

Lane, D., Maxfield, R., 2005. Ontological uncertainty and innovation. Journal of Evolutionary Economics 15, 3-50.

Lane, D., van der Leeuw, S., Sigaloff, C., Addarii, F., 2011. Innovation, sustainability and ICT. FET11 Elsevier Ltd. Selection.

Lane, D. A., 2006. Hierarchy, Complexity, Society. In: Pumain, D. (Ed.), Hierarchy in Natural and Social Sciences. Springer, pp. 81-120.

Lane, D. A., 2011. Complexity and Innovation Dynamics. In: Antonelli, C. (Ed.), Handbook on the Economic Complexity of Technological Change. Edward Elgar Publishing, Cheltenham, Ch. 2, pp. 63-80.

Latour, B., 1993. We Have Never Been Modern. Harvard University Press, Cambridge, MA.

Leach, M., Scoones, I., Stirling, A., 2007. Pathways to Sustainability: an overview of the STEPS Centre approach.

Leach, M., Scoones, I., Stirling, A., Aug. 2010. Governing epidemics in an age of complexity: Narratives, politics and pathways to sustainability. Global Environmental Change 20 (3), 369-377.

Loorbach, D., Jan. 2010. Transition Management for Sustainable Development: A Prescriptive, Complexity-Based Governance Framework. Governance 23 (1), 161-183. 
Malerba, F., 2005. Industrial dynamics and innovation : progress and challenges. In: Presidential address delivered at the 32nd Conference of the European Association for Research in Industrial Economics (EARIE).

Miller, R., May 2007. Futures literacy: A hybrid strategic scenario method. Futures 39 (4), 341-362.

Morin, E., 2007. Restricted complexity, general complexity. ..., science and us: Philosophy and complexity. ..., 5-29.

Odling-Smee, F. J., Laland, K. N., Feldman, M. W., 2003. Niche construction: the neglected process in evolution; Monographs in Population Biology 37. Princeton: Princeton University Press.

Ostrom, E., Sep. 2007. A diagnostic approach for going beyond panaceas. Proceedings of the National Academy of Sciences of the United States of America 104 (39), 15181-7.

Ott, E., 2002. Chaos in Dynamical Systems, 2nd Edition. Cambridge: Cambridge University Press.

Oyama, S., Griffiths, P. E., Gray, R. D., 2001. Cycles of Contingency. MIT Press, Cambridge MA.

Pahl-Wostl, C., 2002. Agent based simulation in integrated assessment and resources management. International Environmental Modelling and Software Society (iEMSs 2002) (2), 239-244.

Pigliucci, M., Müller, G. B., 2010. Evolution, the extended synthesis. MIT Press, Cambridge, MA.

Prigogine, I., Nicolis, G., 1977. Self-Organization in Nonequilibrium Systems : From Dissipative Structures to Order through Fluctuations. John Wiley and Sons.

Ragin, C. C., 2009. Reflections on casing and case oriented research. In: Byrne, D., Ragin, C. C. (Eds.), The SAGE handbook of Case Based Methods. Sage Publications, London, pp. 522-534.

Read, D. W., 2008. Working Memory: A Cognitive Limit to Non-Human Primate Recursive Thinking Prior to Hominid Evolution. Evolutionary Psychology $6(4), 676-714$.

Reed, M., Harvey, D. L., 1992. The new science and the old: complexity and realism in the social sciences. Journal for the Theory of Social Behaviour 22 (4), 353-380.

Rittel, H., Webber, M., 1973. Dilemmas in a general theory of planning. Policy sciences 4, 155-169. 
Rotmans, J., Asselt, M. V., 2003. Integrated Assessment Modelling. In: Climate Change: An Integrated Perspective. Springer Netherlands, Ch. 7, pp. 239275 .

Sawyer, R. K., 2005. Social Emergence: Societies As Complex Systems. Cambridge University Press, Cambridge UK.

Schelling, T. C., 1978. Micromotives and Macrobehavior. W. W. Norton and Co.

Schlosser, G., 1998. Self-re-production and functionality: a systems-theoretical approach to teleological explanation. Synthese 116, 303-354.

Scoones, I., Leach, M., Smith, A., Stagl, S., 2007. Dynamic systems and the challenge of sustainability.

Shalizi, C. R., 2008. Self-organization. http://cscs.umich.edu/〜 crshalizi/notebooks/selforganization.html.

Simon, H. A., 1996. The Sciences of the Artificial, 3rd Edition. MIT Press, Cambridge MA.

Squazzoni, F., Sep. 2008. A (computational) social science perspective on societal transitions. Computational and Mathematical Organization Theory 14 (4), 266-282.

Stewart, P., 2001. Complexity Theories, Social Theory, and the Question of Social Complexity. Philosophy of the Social Sciences 31, 323-360.

Vasileiadou, E., Safarzyska, K., Dec. 2010. Transitions: Taking complexity seriously. Futures 42 (10), 1176-1186.

Walby, S., Dec. 2007. Complexity Theory, Systems Theory, and Multiple Intersecting Social Inequalities. Philosophy of the Social Sciences 37 (4), 449-470.

Weinberg, G. M., 2001. Introduction to General Systems Thinking, Silver Anniversary Edition (originally published 1975). Dorset House.

West Churchman, C., 1967. Guest Editorial. Management Science 14 (4), 141142.

Whiten, A., Byrne, R. W., 1997. Machiavellian Intelligence II. Cambridge University Press, Cambridge.

Whiten, A., van Schaik, C. P., Apr. 2007. The evolution of animal 'cultures' and social intelligence. Philosophical transactions of the Royal Society of London. Series B, Biological sciences 362 (1480), 603-20.

Wimsatt, W. C., 1975. Complexity and organization. In: Topics in the philosophy of biology. Springer, pp. 174-193. 
Wimsatt, W. C., 2007. Re-Engineering Philosophy for Limited Beings: Piecewise Approximations to Reality. Harvard University Press.

Wimsatt, W. C., Griesemer, J. R., 2007. Reproducing Entrenchments to Scaffold Culture: The Central Role of Development in Cultural Evolution. In: Sansom, R., Brandon, R. N. (Eds.), Integrating Evolution and Development: From Theory to Practice. MIT Press, Cambridge, MA, Ch. 7, pp. 227-323. 